\section{Practising sound medicine in the absence of evidence}

$\mathrm{T}$ he commentary by Harriet MacMillan and Nadine Wathen ${ }^{1}$ illustrates some of the problems that may arise in using only an evidence-based approach to guide clinical decisionmaking, rather than balancing existing evidence with clinical judgement. Although it was perhaps not the authors' intention, we are concerned that the message that physicians may take from this article is that they should not screen for abuse because evidence for such an intervention is inadequate.

There is a marked paucity of research in this field, particularly given the pervasiveness of abuse and the injuries and deaths it causes. If we are to use only those interventions for which significant (statistically or otherwise) research has been done, then we may be systematically excluding interventions for which there has been less academic interest and consequently less published research on which to base recommendations.

The suggestion to screen people with signs and symptoms of "potential abuse" is confusing. Although research has been done on the prevalence of various signs and symptoms in people who have been abused, ${ }^{3}$ the predictive value of signs and symptoms has not been high, ${ }^{4,5}$ which suggests that they are not sensitive indicators of abuse. This, coupled with the high prevalence of abuse, justifies universal screening: if, as the authors state, it is appropriate to screen people exhibiting signs and symptoms, then it should be appropriate to screen everyone.

Finally, it appears that the authors did not consider that the act of disclosing to a health care provider an experience of abuse may be a positive out- come in and of itself, if the disclosure is beneficial psychologically.

Given the apparent lack of harm in screening patients for abuse and its potential benefits, which have yet to be adequately investigated, we feel that this intervention should continue to be widely used until further research demonstrates that it is inappropriate or unnecessary.

\section{Fiona Kouyoumdjian \\ Vanessa L. Cardy \\ Medical Students \\ Dalhousie University \\ Halifax, NS}

\section{References}

1. MacMillan HL, Wathen CN. Violence against women: integrating the evidence into clinical practice [editorial]. CMAF 2003;169(6):570-1.

2. Wathen CN, MacMillan HL, with the Canadian Task Force on Preventive Health Care. Prevention of violence against women. Recommendation statement from the Canadian Task Force on Preventive Health Care. CMA7 2003;169(6):582-4.

3. McCauley J, Kern DE, Kolodner K, Dill L, Schroeder AF, DeChant HK, et al. The "battering syndrome": prevalence and clinical characteristics of domestic violence in primary care internal medicine practices. Ann Intern Med 1995; 123(10):737-46

4. Wasson JH, Jette AM, Anderson J, Johnson DJ, Nelson EC, Kilo CM. Routine, single-item screening to identify abusive relationships in women. F Fam Pract 2000;49(11):1017-22.

5. Saunders DG, Hamberger LK, Hovey M. Indicators of woman abuse based on a chart review at a family practice center. Arch Fam Med 1993;2 (5):537-43.

DOI:10.1053/cmaj.1031733

\section{[The authors respond:]}

$\mathrm{W}$ e agree with Fiona Kouyoumdjian and Vanessa Cardy that in the face of uncertain evidence, clinical context and clinician experience will ultimately determine the course of care. Unfortunately, Kouyoumdjian and Cardy seem to have misunderstood a key point of our commentary ${ }^{1}$ and the accompanying recommendations of the Canadian Task Force on Preventive
Health Care. ${ }^{2}$ We do not recommend that clinicians "screen people with signs and symptoms of "potential abuse"; rather, good clinical care demands accurate diagnosis and appropriate treatment when a woman presents with injuries or other manifestations consistent with abuse. The difficulty in recommending universal screening - that is, routine assessment of all women presenting for any medical concern - is the lack of evidence regarding appropriate treatment interventions.

Kouyoumdjian and Cardy further state that there is an "apparent lack of harm in screening patients for abuse" and that the act of disclosing abuse "may be a positive outcome in and of itself." In fact, a range of potential harms may result from screening, including the possibility of psychological distress (as opposed to the benefit assumed by Kouyoumdjian and Cardy) when a woman is asked to disclose abuse when she is not ready to do so; ${ }^{4}$ the raising of false hope that screening can help, when in fact it may not; and the potential of exposing the woman to further violence. The lack of evaluation of the potential harms of screening is a major problem in this field. No intervention is completely without harm or cost, whether it be opportunity cost (e.g., the clinical time required for screening that could be spent on other problems) or a specific risk associated with the intervention or its sequelae (e.g., adverse reaction to a vaccine). It is essential that screening be evaluated to determine whether it does more good than harm, rather than simply assuming that it has benefit. As outlined in our commentary, ${ }^{1}$ we are fortunate that various organizations, including the US Centers for Disease Control and Prevention and the Ontario 
Women's Health Council, have recognized this gap in scientific evidence and that studies to address this question are under way.

\section{Harriet L. MacMillan \\ Departments of Psychiatry and \\ Behavioural Neurosciences and of \\ Pediatrics \\ C. Nadine Wathen \\ Offord Centre for Child Studies \\ McMaster University \\ Hamilton, Ont.}

\section{References}

1. MacMillan HL, Wathen CN. Violence against women: integrating the evidence into clinical practice [editorial]. CMA7 2003;169(6):570-1.

2. Wathen CN, MacMillan HL, with the Canadian Task Force on Preventive Health Care. Prevention of violence against women. Recommendation statement from the Canadian Task Force on Preventive Health Care. CMA7 2003;169(6):582-4.

3. Wathen CN, MacMillan HL. Interventions for violence against women: scientific review. $7 A M A$ 2003;289:589-600,e1-e10.

4. Chang JC, Decker M, Moracco KE, Martin SL, Petersen R, Frasier PY. What happens when health care providers ask about intimate partner violence? A description of consequences from the perspectives of female survivors. $7 \mathrm{Am} \mathrm{Med}$ Womens Assoc 2003;58:76-81.

DOI:10.1053/cmaj.1031877

\section{The high impact of an influenza pandemic}

$I^{2}$ pplaud the CMAF for its efforts to increase public and political awareness of the potential impact of an influenza pandemic in Canada. However, I would like to clarify one of the numbers that appeared in a recent news article. ${ }^{1}$

It is difficult to precisely quantify or to exaggerate - the impact of the next influenza pandemic. That impact will depend on how virulent the virus is, how rapidly it spreads from person to person, and how effective and available prevention and control measures prove to be. Estimates based on previous pandemics (in 1918, 1957 and 1968) can be used as a guide, but global travel is far greater than ever before and will no doubt accelerate the speed of international spread, as with SARS.

Models have been developed to estimate the possible impacts of the next pandemic, ${ }^{2}$ but they are based on as- sumptions derived from the US experience, and their applicability to other health care settings or systems is limited. Nor do these models incorporate the use of antivirals or vaccines, should these become sufficiently available.

Estimates of impact can nevertheless be useful in showing the scale or magnitude of the crisis relative to that of other disasters and in increasing the awareness that is critical to preparation and planning required to minimize that impact. Assuming attack rates in the range of $15 \%$ to $35 \%$ during the next influenza pandemic, the Canadian Pandemic Influenza Plan (www.hc-sc.gc.ca /pphb-dgspsp/cpip-pclcpi/) incorporates the Meltzer model in estimating that 5-10 million Canadians could become clinically ill, such that they would be unable to attend work or other activities for at least half a day. Furthermore, an estimated 2-5 million Canadians would require outpatient care, between 30000 and 140000 would require admission to hospital, and between 10000 and 60000 could die. As such, although it is staggering to imagine and difficult to accept, the estimate of 50000 cited in the CMAF article refers not to the number in Canada who could become ill but to the number who could ultimately perish.

Because communities would be affected over a short period of time (6-8 weeks), simultaneously and possibly in 2 waves during the same season, a pandemic of influenza will be unlike any other catastrophe. The SARS outbreak was an important but limited rehearsal that has helped further refine our preparedness. Technology has improved substantially since avian influenza H5N1 first appeared among humans in 1997 , and our ability to detect and respond to this virus and its pandemic potential is far better. Either way, it is clear that a pandemic of influenza has the potential to be exponentially worse than SARS in its capacity to cause human suffering - both illness and despair - let alone economic and social upheaval.

Given the warning signals repeatedly emanating from Southeast Asia, an all-out and unified international effort to mitigate this possibility should be undertaken now.

\section{Danuta Skowronski}

Epidemiology Services

BC Centre for Disease Control Society

Vancouver, BC

\section{References}

1. Andresen M. "Imminent" flu pandemic: Are we ready? CMA7 2004;170(2):181.

2. Meltzer M, Shoemake H, Kownaski M, Crosby R, Smith D. FluAid 2.0: Estimating the state level impact of pandemic influenza. Version 2.0 [manual and computer program]. Atlanta: Centers for Disease Control and Prevention; 2000. Available: www2a.cdc.gov/od/fluaid/ (accessed 2004 Feb 3).

DOI:10.1053/cmaj.1040167

\section{Direct-to-consumer advertising}

$\mathrm{B}$ arbara Mintzes and colleagues ${ }^{1}$ seem to demonstrate that directto-consumer advertising (DTCA) has net benefits, despite their own pessimistic interpretation. Patients who requested advertised drugs received them in $86.5 \%$ of cases, whereas only $26.2 \%$ of patients who did not make a request received a prescription. However, $74.3 \%$ of patients who requested nonadvertised drugs received prescriptions. Thus, although DTCA was associated with a greater proportion of requests that were fulfilled, the effect was minor relative to other, unexplained, reasons for requesting prescriptions.

For prescriptions that were given without being requested, physicians considered only $12.1 \%$ "possibly" or "unlikely" to be appropriate for similar patients. However, for requested prescriptions for advertised drugs, the share was $50.0 \%$, and for requested prescriptions for nonadvertised drugs it was $39.6 \%$. Thus, the same principle applies here as above.

Of prescriptions for advertised drugs, half were unambiguous (i.e., were not associated with physician ambivalence about appropriateness), and it is not stated how many, if any, of these ambiguous prescriptions had negative outcomes. I have shown elsewhere ${ }^{2}$ that if less than $42 \%$ of ambiguous prescrip- 